Die Zeit, die zur Umwandlung der Gallerte in kristallisiertes Salz erforderlich ist, wechselt stark mit der Gallertmenge und der Art der Erhitzung; bei größeren Gallertmengen (mit geringem UeberschuB an Arsenatlösung) ist die Umwandlungszeit wesentlich kürzer als bei den Gallertmengen der in Tabelle II angegebenen Versuche.

\section{Einflub des Wasserzusatzes a die Gallertbildung.}

Die Versuche wurden so ausgeführt, daß in einem Becherglas $10 \mathrm{ccm}$ Manganlösung mit wechselnden Mengen Wasser vermischt wurden; zur Mischung wurden hierauf $20 \mathrm{ccm}$ der Arsenatlösung rasch hinzugegeben. Sämtliche Lösungen besaßen Zimmertemperatur. Die Mischungen blieben zur Beobachtung stehen; durch zeitweiliges Neigen des Becherglases wurde das Voranschreiten der Gallertbildung, so gut als dies möglich ist, beobachtet.

Tabelle ill

\begin{tabular}{|c|c|c|c|}
\hline $\begin{array}{c}\text { Versuch } \\
\mathrm{Nr} . \\
\end{array}$ & $\begin{array}{c}\text { Zugesetzte } \\
\text { Wassermenge } \\
\mathrm{ccm}\end{array}$ & $\begin{array}{l}\text { Zeit, nach welcher } \\
\text { feste Gallerte be- } \\
\text { obachtet wurde }\end{array}$ & $\begin{array}{l}\text { Aussehen } \\
\text { der } \\
\text { Gallerte }\end{array}$ \\
\hline $\begin{array}{r}1 \\
-\quad 2\end{array}$ & $\overline{5}$ & $\begin{array}{ccc}\text { etwa } & 8 / 4 \\
" & \text { Min. }\end{array}$ & $\begin{array}{l}\text { truibe } \\
\text { schwächer }\end{array}$ \\
\hline $\begin{array}{l}3 \\
4\end{array}$ & $\begin{array}{l}10 \\
15\end{array}$ & $\begin{array}{lll}\prime & 2 & \\
" & 4 & \end{array}$ & $\begin{array}{l}\text { opalisierend } \\
\text { schwach }\end{array}$ \\
\hline $\begin{array}{l}5 \\
6 \\
7\end{array}$ & $\begin{array}{l}20 \\
30 \\
40\end{array}$ & $\begin{array}{c}" 10 \\
30 \\
\text { nach } 24 \text { Std. "noch }\end{array}$ & $\begin{array}{l}\text { fast klar } \\
\text { klar } \\
\text { do. }\end{array}$ \\
\hline 8 & 50 & $\begin{array}{l}\text { nach } 24 \text { Std. } \\
\text { noch gröistenteils }\end{array}$ & do. \\
\hline 9 & 100 & $\begin{array}{l}\text { nach } 24 \text { Std. } \\
\text { flüssig }\end{array}$ & do. \\
\hline
\end{tabular}

Die Beobachtungen sind tn Tabelle IIl zusammengestellt.

Da bei den Mischungen mit höherem Wasserzusatz (Versuche 7-9) die Gallertbildung nur in geringem Maße oder gar nicht bemerkbar war, so wurde bei diesen Versuchen das Becherglas mit der Mischung nachträglich zwei bis drei Minuten lang in das Dampfbad eingehängt, so daß die ganze Flüssigkeit gleichmäBig erwärmt wurdé. Beim Hetausnehmen der Becherg!äser zeigte sich, daß bei sämtlichen Mischungen Gallertbildung eingetreten war; selbst die Gallerte des Versuches 9 war dabei so fest geworden, dabi sie beim Neigen des Becherglases sich nicht mehr bewegte. Beim Erkalten der Gallerten war keine Verflüssigung zu beobachten.

\section{Zusammenfassung.}

In Vorstehendem ist gezeigt:

1. Die Umsetzung zwischen primärem Alkaliarsenat und Manganosalz führt bei hinreichender Konzentration der Lösungen zur Bildung einer rein anorganischen Gallerte. Die Zusammensetzung der Gallerte entspricht der des sekundären Manganoarsenates $\mathrm{MnHAsO}_{4}$.

2. Die Gallerte kann in kristallisiertes Manganoarsenat der Zusammensetzung

$$
\mathrm{MnHAsO} \mathrm{O}_{4}+\mathrm{H}_{2} \mathrm{O}
$$

übergeführt werden. Bei langem Stehenlassen der Gallerte in Zimmerwärme kann man die Bildung kugelförmiger Kristallgebilde beobachten; beim Erwärmen der Gallerte findet raschere Kristallisation statt:

3. Das durch Erwärmen der Gallerte erhaltene kristallisierte Manganoarsenat bildet glänzende violettrosa gefärbte Blättchen, die in Wasser schwer löslich sind.

Berlin-Steglitz, im Dezember 1913.

\title{
Ueber Meerschaum von Grant Co. in Neu-Mexiko
}

\author{
Von H. Michel (Wien).
}

(Eingegangen sin 31. Januar 1914)

Im Anschluf an meine früheren Bemerkungen über Meerschaumn ${ }^{1}$ möchte ich über ein Vorkommen von Meerschaum in NeuMexiko berichten, das von der Foote Mineral Company in Philadelphia vertrieben wird. Ich verdanke Proben desselben dem $K$. $K$. naturhistorischen Hofnuseum in Wien durch die Liebenswürdigkeit des Herrn Dr. Koechlin.
Von B. Sterett ${ }^{2}$ ) rührt eine vom technischen Standpunkt abgefaßte Beschreibung dieses Meerschaumvorkommens her; es sind zwei räumlich getrennte Vorkommen in Neu-Mexiko vorhanden, die beide analysiert wurden. Das eine Vorkommen von Dorsey Mine hat die Zusammensetzung eines Meerschaumes, das andere von Sapillo Creek weicht sehr stark

9) B. Ste r ett, U.St. Geol. Bulletin 340,467 (1908).

1) Koll.-Zeitschr. 12, 165 (1913). 
von der Zusammensetzang eines Meerschaums $a b$, wie die folgenden Zahlen zeigen:

\begin{tabular}{|c|c|c|}
\hline & $\begin{array}{c}\text { l } \\
\text { Dorsey Mine }\end{array}$ & $\stackrel{11}{\text { Sapillo Creek }}$ \\
\hline $\begin{array}{c}\mathrm{SiO}_{2} \\
\mathrm{Al}_{2} \mathrm{O}_{5} \\
\mathrm{FeO}_{3} \\
\mathrm{MgO} \\
\mathrm{CaO} \\
\mathrm{CO}_{2} \\
\mathrm{H}_{2} \mathrm{O}\end{array}$ & $\begin{array}{r}57,10 \\
0,58 \\
\text { Spur } \\
27,16 \\
0,17 \\
0,32 \\
14,78 \\
100,1 !\end{array}$ & $\begin{array}{r}60,97 \\
19,71 \\
10,00 \\
0,22 \\
19,14 \\
100,04\end{array}$ \\
\hline
\end{tabular}

Die Analyse I rührt von G. Steiger her, die Analyse II von W. T. Schaller, beide werden von $B$. Steret t zitjert.

Das Stïck, welches dem Verfasser zur Verfïgung stand, stammt von Dorsey Mine, wie die Analyse ergab. Bei der Untersuchung von Dünnschliffen zeigt sich, daß hier ein nahezu yanz kristallines Produkt volliegt. Bei einer früheren Gelegenheit ${ }^{3}$ ) hat der Verfasser bezinglich des Meerschaumvorkommens von Klein. asien die Ansicht ausgesprochen, dab sie Gemènge eines kristallinen Faserminerales mit einem Gele sind und daf dieses kristalline Fasermineral nach seinen optischen Eigenschaften ident sein könnte mit dem von $A$. Fersmann als $P$ at as epiolith bezeichneten Meerschaum, dem dic Formel $\mathrm{H}_{4} \mathrm{Mg}_{2} \mathrm{Si}_{3} \mathrm{O}_{10} .2 \mathrm{H}_{2} \mathrm{O}$ zugeschrieben wird. $O \mathrm{~h}$ der krisialline Anteil diese lusammensetzung hat, konnte nicht entschieden werde!n.

Da nun dieses mexikanische Vorkommen das kristalline Fasermineral in großer Reinheit and mit weit geringeren Beimengungen von kolloider Substanz zeigte, schien eine genauere Untersuchung wïnschenswert.

Bereits makroskopisch ist das Auftreten von Blasenräumen in der schmeeweißen und sonst ganz dichten Masse zu bemerken, die von einem duchscheinenden, etwas milchig weib opaleszierenden, ganz drimnen Häutchen ansgekleidet werden und sich mit gröRter leichtigkeit eindrücken lassen. Ist das dünne Häıtchen gespanut, so erweckt es nicht den Eindruck einer Hohlraumumkleidung, sondern man glaubt, es seien in der dichten faserigen Hauptuasse milchopalartige Partien eingesprengt.

Bei der mikrosliopischen Prüfung dieser Häntchen zeigt sich, daß sie zum gröbten Teil tatsilchlich aus ciner kolloiden Substane be-

i) H. Miche 1. Koll-Zeitschr. 12, 165 (1913). Daselbst auch die einsionlägige Literatur. stehen, in welcher kristalline Fäserchen eirgebettet erscheinen. Diese Fäserchen sind schwächer lichtbrechend als die kolicide Sirstan\%, die Brechungsexponenten liegen ganz in der Nahe des Brechungsquotienten von reinem Berizol $(\mathrm{n}=1,5016$ ist der Brechungsqunien! des verwendeten Benzols). Die kolloide Substan\% hat sinen Brechungsquotienten von 1.5159 bei Zimmertemperatur und initterer fenchtig. keit der Luft. Die Fasern lüschen gerade aus, für thre Lingsrichtung gilt der gröfere Brechungsquotient.

Ganz das gleiche Fasermineral findet sich 'in der kompakten Masse, in der die Bläschen auftreten. Hier überwirgt jedoch das Fasermineral, das Kolloid tritt sehr stark zhinick. Hicr sind die Fasern wuch besser ausgebildet, so dak sie genauer geprüft werden können. Auch hier hat das Kolloid eine etwas hoblere Lichtbrechung, lee 1,520, während die Brecaungsquotienten des Faserminerals zwischen 1,500 und 1,512 liegen. Die Doppelbrechung ergibt sich nach der Tabelle von H. Rosenbusch mit $\gamma \ldots=0,010$ bis 0,012 In de Längsrichtung schwingt der langsamere Strahl, die Auslöschung ist gerade. Bei den kieinasiatischen Vorkommen schien det Achsenwinkel um a gegen $50^{\circ} \mathrm{zu}$ betragen, hier ist er sicher gröber, so daB eine sichere Entscheidung, ob positive oder negative Doppelbrechung vorliegt, nicht getroffen werden konnte. Miog licherweise ändert sich der Achsenwinkel mit dem Wassergehalt, da anch die Brechungs. exponenten hier etwas niednger sind, tis be: den kleinasiatischen Vorkommen. Das Wassin scheint teilweise $\left(2 \mathrm{H}_{2} \mathrm{O}\right)$ nach Art des Zeolithwassers gebunden zu sein.

Bemerkenswert ist es, dab die vom Verfasser früher unte!suchten Vorkommen etwas höhere Brechungsq:otienten zeigten und eine größere Menge kolloider Substanz beigemengt enthielten. Es kön :te hier das Kolloid was: entzichend wirken und einen Teil des locker gebundenen Wassers absorbieren.

Bei der Anfärbung mit sauren und basischen Farbstoffen tritt stets eine Absorption des Farbstoffes ein. Wird das Ehrlich'sche Triazid-Gemisch basisches Methylenblau .. Säurefuchsin verwendet, so firbt sich das Kolloid, obwohl es nur in sehr geringer Menge vor. handen ist, sofont und interisiy blan an, zeigt also basophilen Charakter. Das kristalline Fasermineral ist in derseiben Zeit überhaupt nicht anfärbbar, es iäBt sich der Farbsioff, nachdem er einige Minuten eingewirkt hat, 
leicht wieder auswaschen, während dies beim kolloiden Anteil nicht der Fall ist. Erst nach mehrstündiger Einwirkung läbt sich an dem ausgewaschenen Pulver eine ganz blasse Rosafärbung kristalliner Anteile feststellen.

Während der Verfasser geneigt war, diese blasse Rosafärbung als schwache Oxyphilie der kristallinen Anteile zu deuten, im Einklang mit der deutlich basischen Reaktion des Meerschaums, glaubt $F$. Hundeshagen, mit welchem Verfasser tiber diesen Gegenstand einen Briefwechsel führte, an eine Imbibitionsfärbung, die nach dem Auswaschen zurückbleibt und keine "typische oxyphile" Färbung wäre. $\mathrm{Da}$ jede kristalline Faser von kolloider Substanz umhüllt wird, die den basischen Farbstoff stark absorbiert, ist diese Ansicht nicht von der Hand $z u$ weisen. Jedenfalls verhalt sich aber der kristalline Anteil verschieden vom kolloiden Anteil, und in dieser Hinsicht ist es gleichgültig, ob die kristallinen Fasern oxyphil sind oder $o b$ sie durch Imbibition rot gefärbt sind, da ja nur die Feststellung von Wichtigkeit ist, dab im Meerschaum ein kolloider und ein kristalliner Anteil vorliegen.

In geglühtem Zustande nimmt das Pulver nur äuberst wenig vom Farbstoff auf, es verliert seine Anfäbbarkeit.

Die oben erwähnten, zum größten Teil aus kolloider Substanz bestehenden Hautchen färben sich rasch und intensiv blau an, die Färbung der darin enthaltenen kristallinen Fäserchen wird ganz überdeckt.

Das Pulver des Meerschaums reagiert deutlich basisch.
Nach dem Glühen des Meerschaumpulvers tritt eine dauernde Veränderung ein, indem sich Lichtbrechung und Doppelbrechung ändert. Die Brechungsquotienten liegen nunmehr bei $\gamma=1,539-1,538$ und $\alpha=1,530$; die Doppelbreching ist etwas schwächer. Auch nach achttägigem Stehen bleiben die Brechungs quotienten in dieser Höhe. Die Interferenzbilder erscheinen gestört.

In physikalischer Hinsicht stimmt also das Fasermineral mit dem Parasepiolith überein; um auch chemische Uebereinstimmung nachzuweisen, wurde eine Analyse ausgeführt, wobei darauf verzichtet wurde, das Kolloid von dem kristallinen Anteil zu trennen, da die Menge des Kolloides ohnedies sehr gering ist.

Die Analyse ergab folgende Zahlen:

\begin{tabular}{c|c|c|c}
\hline & \multicolumn{1}{|c|}{$\mathrm{I}$} & II & III \\
\hline $\mathrm{SiO}_{2}$ & 54,76 & 0,90812 & 54,23 \\
$\mathrm{Fe}_{2} \mathrm{O}_{3}$ & $\mathrm{Spur}$ & & - \\
$\mathrm{Al}_{2} \mathrm{O}_{9}$ & 0,33 & 0,003229 & - \\
$\mathrm{CaO}$ & $\mathrm{Spur}$ & & - \\
$\mathrm{MgO}^{\prime}$ & 25,15 & 0,62376 & 24,17 \\
$\mathrm{H}_{2} \mathrm{O}$ unter $110^{\circ}$ & 10,46 & 0,5806 & 10,80 \\
$\mathrm{H}_{2} \mathrm{O}$ iber 1100 & 9,71 & 0,53897 & 10,80 \\
Summe & 100,41 & & 100,00
\end{tabular}

Unter Il sind die Molekularquotienten angeführt, unter III die theoretische Zusammensetzung des Parasepiolithes; setzt man den Molekularquotienten für $\mathrm{SiO}_{2}=3$, so erhält man folgendes Molekularverhältnis der einzelnen Bestandteile zueinander:

$$
\begin{gathered}
\mathrm{SiO}_{2}: \mathrm{Al}_{2} \mathrm{O}_{2}: \mathrm{MgO}: \mathrm{H}_{2} \mathrm{O} \text { unter } 1000: \mathrm{H}_{2} \mathrm{O} \text { über } 100^{\circ} \\
\text { wie } 3: 0,011: 2,056: 1,918: 1,780
\end{gathered}
$$

Dieses Verhältnis entspricht recht gut der Formel des Parasepiolithes $3 \mathrm{SiO}_{2} .2 \mathrm{Mg} \mathrm{O} .4 \mathrm{H}_{2} \mathrm{O}$. Das von A. Farsmann ${ }^{4}$ als typischer Parasepiolith bezeichnete Vorkommen von Tempelstein ergab nach der Analyse von F. Kovát ${ }^{3}$ ) das Verhälnis :

$$
\begin{gathered}
3 \mathrm{SiO}_{2}: 0,08 \mathrm{Al}_{2} \mathrm{O}_{3} \\
: 0,02 \mathrm{Fe}_{2} \mathrm{O}_{\mathrm{a}}: 2,03 \mathrm{R}^{11} \mathrm{O}: 3,69 \mathrm{H}_{4} \mathrm{O}
\end{gathered}
$$

Es ist also grobe Wahrscheinlichkeit vorhandex, dab das kristalline Silikat, welches in unserem Falle nahezu allein vorhanden ist -das beigemengte Kolloid tritt an Menge sebr

4) Fersman a. SB. d. böhmi. Akad. 1912.

5) F. Ková . Programm deta cechosl. Handelsak. Prag 1913. stark zurück - dieser Pormel tatsächlich entspricht und also mit dem Parasepiolith identisch ist. Nur der Wassergehalt weicht etwas stärker von der theoretisch geforderten Menge $a b$, weil sich eben gerade im Wassergehalt eine auch noch so geringe Menge eines beigemengten Gels am stärksten bemerkbar zu machen vermag.

W. Vernadsky ${ }^{6}$ ) hatte bereits fräher in der Meerschaumgruppe eine Zweiteilung vorgenommen und $\alpha$-Sepiolithe und $\boldsymbol{\beta}$-Sepiolithe unierschieden. Erstere geben mit Säuren eine gallertige Ausscheidung, letztere' nicht, erstere sind wasserreicher. Vernadsky schrieb dem $\alpha$-Sepiolith dieselbe Formel zu, die Fers-

6) W. Vernads ky, Zeitschr. t. Krist. 34, 46 (1901). 
mann fur den Parasepiolith annimmt und die auch im vorliegenden Falle wahrscheinlich ist. Der $\alpha$-Sepiolith hat nach Vernads $k$ y folgende Strukturformel:

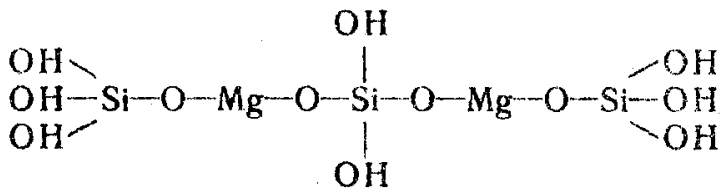

Die $\beta$-Sepiolithe, welche weniger Wasser enthalten, haben nach $V \in r n a d s k y$ die $Z u-$ sammensetzung $\mathrm{Mg}_{2} \mathrm{H}_{4} \mathrm{Si}_{3} \mathrm{O}_{10}$ und die Strukturformel:<smiles>O=[Si](O)O[14CH2]O[Si](O)(O)O[As]O[Si](=O)O</smiles>

Mit Salzsäure gibt der Meerschaum von Grant Co. eine Gallerte; die Abscheidung derselben erfolgt leichter als bei den vom Ver* fasser früher beschriebenen kleinasiatischen Vorkommen.

Es stimmt also das kristalline Fasermineral, welches in dem hier beschriebenen Meerschaum in überwiegender Menge vorhanden ist, in allen Punkien gut mit dem $\alpha$-Sepiolith Vernadsky's und dem Parasepiolith yon A. Fers mann überein, so dab es wohl mit diesem ident ist; lediglich der Wassergehalt schwankt etwas stärker. Wenn man jedoch die Formel des Parasepiolthes nach $A$. Fersman

$$
\mathrm{H}_{1} \mathrm{Mg}_{2} \mathrm{Sig}_{3} \mathrm{O}_{10} \cdot 2 \mathrm{H}_{2} \mathrm{O}
$$

schreibt und annimmt, da $2 \mathrm{H}_{2} \mathrm{O}$ etwa mach Art des Zeolithwassers gebunden sind, so ist auch das leicht erklärlich.

Jedenfalls darf man dem Fasermineral die Formel des Parasepiolithes zuschreiben und in den Meerschaumvorkommen ein Gemenge des Parasepiolithes mit einer kolloiden Substanz annehmen.

\section{Mineralogisches Institut der Universität Wien.}

\section{Die zeitliche Hydrolyse als kolloidchemischer Vorgang.}

\section{Von Carl L. Wagner.}

(Eingegangen am 15. Jauuar 1014)

(Aus dem physikalisch-chemischen Institut der Deutschen Universitat Prag.)

Ich habe vor einiger Zeit zur Prüfung einer besonderen Vorstellung, die ich mir von dem Zustandekommen der sogenannten "zeitlichen Hydrolyse" gebildet hatte, eine Reihe von Versuchen angestellt über die Aenderung des elektrischen Leityermögens mancher Salze mit der Zeit und habe deren bisherige Ergebnisse an anderer Stelle) ausführlich berichtet. Da diese Arbeiten eine Unterbrechung erfuhren, so folge ich sehr gern einer freundlichen. Aufforderung des Herrn Herausgebers dieser Zeitschrift, eine Zusammenfassung meiner diesbezüglichen Untersuchungen mit Betonung der kolloidchemischen Seite zu geben.

Bekanntlich kann der hydrolytische Zerfall seibst bei Salzen starker Săuren unter Umständen erhebliche Beträge annehmen, wenn die Base sehr schwach ist. Das gleiche gilt von Salzen starker Basen mit sehr schwachen Säuren. In beiden Fällen gibt sich die Hydrolyse durch abnorm hohe Werte der elektrischen Leittähigkeit mit zunehmender Verdünnung kund, da ja schnell wandernde Wasserstoffbzw. Hydroxylionen auftreter. (1913)

1) Monatsh. f. Chem. 34, 95--170 and $931 \cdots-948$
Es gibt nun Salze, deren Hydrolyse lang. sam verläuft. Verdünnt man die konzentrierte Lösung eines solchen Salzes plötzlich sehr stark, so zeigen sic nicht sogleich das ihnen infolge der starken Hydrolyse zukommende große Leitvermögen, sondem dieses steigt allmählich bis zu einem gewissen Endwert. So verhalten sich die Silikate der Alkalien, verschiedene Gold- und Platinsalze, die Ferri- und Stanniverbindungen, ferner Salze des dreiwertigen Chroms, des Zirkons, Titans, Aluminiums und andere.Der zeitlicheVerlaufder Hydrolyse, die bei allen diesen angeführien Salzen oft bequem meBbar verfolgt werden kann, steht im Gegensatz zu den sonst bei lonenreaktionen - und zu diesen müssen wir die Hydrolyse eines Salzes rechnen - beobachteten großen Geschwindigkeit. Hier tritt also der Fall ein, wo anscheinend einmal eine Ionenreaktion nicht, wie man sich gewöhnlich ausdrückt, "unmefbar schnell" stattfindet. Es hat auch nicht an Versuchen gefehlt, eine Erklärung für das allmähliche Ansteigen der leitfăhigkeit zu geben. So wurde von manctien Forschern ${ }^{2}$ )

7) W. Kowalewsky, Zeitschr f. anorg. Chen. $22,1(1900)$. 